\title{
Direct Determination of the Site of Addition of Glucosyl Units to Maltooligosaccharide Acceptors Catalyzed by Maize Starch Synthase I
}

\author{
Ying Xie ${ }^{1,2}$, Adam W. Barb1, Tracie A. Hennen-Bierwagen' and Alan M. Myers ${ }^{1 *}$ \\ ${ }^{1}$ Roy J. Carver Department of Biochemistry, Biophysics, and Molecular Biology, lowa State University, Ames, IA, \\ United States, ${ }^{2}$ College of Agronomy, Sichuan Agricultural University, Chengdu, China
}

Starch synthase (SS) (ADP-glucose:1,4- $\alpha$-D-glucan 4- $\alpha$-D-glucosyltransferase) elongates $\alpha-(1 \rightarrow 4)$-linked linear glucans within plastids to generate the storage polymers that constitute starch granules. Multiple SS classes are conserved throughout the plant kingdom, indicating that each provides a unique function responsible for evolutionary selection. Evidence has been presented arguing for addition of glucosyl units from the ADPglucose donor to either the reducing end or the non-reducing end of

OPEN ACCESS

Edited by:

Michael James Emes,

University of Guelph, Canada

Reviewed by:

lan Joseph Tetlow,

University of Guelph, Canada

Naoko Fujita,

Akita Prefectural University, Japan

*Correspondence:

Alan M. Myers

ammyers@iastate.edu;

albergo@mac.com

Specialty section:

This article was submitted to

Plant Metabolism

and Chemodiversity,

a section of the journal

Frontiers in Plant Science

Received: 11 June 2018

Accepted: 07 August 2018

Published: 31 August 2018

Citation:

Xie Y, Barb AW,

Hennen-Bierwagen TA and Myers AM (2018) Direct Determination of the

Site of Addition of Glucosyl Units to Maltooligosaccharide Acceptors

Catalyzed by Maize Starch Synthase

I. Front. Plant Sci. 9:1252.

doi: 10.3389/fpls.2018.01252 the acceptor substrate, although until recently direct evidence addressing this question was not available. Characterization of newly incorporated glucosyl units determined that recombinant maize (Zea mays L.) SSlla elongates its substrates at the non-reducing end. However, the possibility remained that other SSs might utilize distinct mechanisms, and that one or more of the conserved enzyme classes could elongate acceptors at the reducing end. This study characterized the reaction mechanism of recombinant maize SSI regarding its addition site. Newly incorporated residues were labeled with ${ }^{13} \mathrm{C}$, and reducing ends of the elongation products were labeled by chemical derivitization. Electrospray ionization-tandem mass spectroscopy traced the two parameters, i.e., the newly added residue and the reducing end. The results determined that SSI elongates glucans at the non-reducing end. The study also confirmed previous findings showing recombinant SSI can generate glucans of at least 25 units, that it is active using acceptors as short as maltotriose, that recombinant forms of the enzyme absolutely require an acceptor for activity, and that it is not saturable with maltooligosaccharide acceptor substrates.

Keywords: maize, starch synthase, enzymology, starch, amylopectin metabolism

\section{INTRODUCTION}

Starch synthases (ADP-glucose:1,4- $\alpha$-D-glucan 4- $\alpha$-D-glucosyltransferase) (SS) fulfill a critical function in our biosphere by converting photosynthetically-reduced carbon into storage polymers that subsequently provide chemical energy necessary for plant life cycles and indirectly for animal life cycles as well. These enzymes catalyze the transfer of glucosyl moieties from ADP-glucose (ADPGlc) donors to $\alpha-(1 \rightarrow 4)$-linked glucan polymer acceptors, elongating the polymers by one unit each reaction cycle (Keeling and Myers, 2010). Products of cumulative SS activity, amylopectin and amylose, also include 
$\alpha-(1 \rightarrow 6)$ branch linkages introduced by starch branching enzymes (SBEs). Selected hydrolysis of a subset of branch linkages by starch debranching enzymes (DBEs) is also involved in the amylopectin biosynthetic mechanism (Hennen-Bierwagen et al., 2012). Together this enzyme system generates polymers that adopt a semi-crystalline structure within starch granules, and such higher order organization enables long term photosynthate storage (Jeon et al., 2010). Thus, architectural specificity of SS action, in addition to the chemical reaction itself, is responsible for the important functional role of these enzymes.

Architectural organization of glucan polymers in starch granules involves multiple SS conserved in apparently all chloroplast-containing species, i.e., green algae and land plants. Genes encoding five SS classes are present in each genome examined to date, designated GBSS, SSI, SSII, SSIII, and SSIV (Deschamps et al., 2008; Hennen-Bierwagen and Myers, 2013). In some species gene duplication has resulted in multiple isoforms within a class. Strict evolutionary conservation indicates each SS class likely provides a selected function, some of which are relatively well-understood and others that remain to be determined. GBSS generates long linear polymers of several 100 residues that make up amylose, and SSIV appears to have a particular function in initiating starch granule formation (Szydlowski et al., 2009). SSI, SSII, and SSIII generate linear chains that crystallize to form clusters within amylopectin, although their specific functions and how they impart them at the molecular level are not known (for review see Zeeman et al., 2010; Nakamura, 2015). Genetic analyses revealing effects of eliminating specific SS enzymes have led to models suggesting SSI elongates linear chains created by SBE action, starting at degree of polymerization (DP) 6 or 7, to approximately DP12, then SSII elongates those chains further to a distribution centered about DP20 (Crofts et al., 2017). These chains then crystallize into clusters that are the basis of the higher order starch structures. SSIII is proposed from such studies to generate chains of DP30 and longer that connect clusters (Fujita et al., 2007; Zhu et al., 2013).

Such models, however, do not fully explain SS action in vivo nor the reason that each class is conserved. They are based primarily on findings that eliminating an SS changes the frequency of occurrence of a particular chain length, rather than eliminating formation of such chains. For example, as the general model indicates, loss of SSII in multiple species results in decreased abundance of approximately DP12 to DP20 chains (Zhang et al., 2004 and references therein). Chains in that length range still exist in SSII mutants, however, at approximately $80 \%$ of the normal level. Thus, there must be considerable overlap in the ability of other SS classes to create DP12-DP20 chains. Similarly, loss of SSI, in Arabidopsis leaf or rice endosperm causes decreased frequency of approximately DP8 to DP12, yet chains of those lengths are still present in the mutant amylopectin (Delvallé et al., 2005; Fujita et al., 2006; Crofts et al., 2017). Further, there is little evidence available indicating distinctions in substrate specificities between SS clases. In vitro studies with recombinant enzymes comparing properties of different SS classes found similar reaction rates of SSI and SSII toward linear glucans from DP2 to DP8, and that SSI is capable of elongating to DP40 and longer (Cuesta-Seijo et al., 2015). Thus, there is no obvious mechanistic reason that explains why SSI, SSII, and SSIII each are invariably retained throughout green plant evolution.

A fundamental question regarding SS mechanism is whether addition of glucosyl residues occurs at the reducing- or nonreducing end of the acceptor chain. A non-reducing end mechanism was long accepted in the literature, based on findings that newly incorporated labeled residues are released by exohydrolases known to proceed from that terminus of linear glucans (De Fekete et al., 1960; Leloir et al., 1961; Recondo and Leloir, 1961). Such a mechanism would enable rapid growth of branched amylopectin precursors, because such molecules have only a single reducing end compared to 1000s of nonreducing ends. More recently, reducing end addition by SSs was proposed based on the observation that newly incorporated glucosyl residues can be chemically derivatized in reactions that require free aldehyde groups (Mukerjea et al., 2002; Mukerjea and Robyt, 2005a,b, 2012).

Direct determination of the site of addition of new glucosyl residues has been described in only one instance. Recombinant SSIIa from maize was used to elongate maltohexaose, using ADPGlc containing uniformly ${ }^{13} \mathrm{C}$-labeled glucosyl units (ADP- $\left.\left[{ }^{13} \mathrm{C}_{\mathrm{U}}\right]-\mathrm{Glc}\right)$. Newly incorporated residues could thus be distinguished from pre-existing residues by a mass difference of $6 \mathrm{Da}$. After the polymers were elongated by SSIIa, they were labeled by reductive amination with $N$-naphthyl-ethylene diamine ( $N$-EDA), adding a defined mass label specifically to the reducing end residue. Electrospray ionization-tandem mass spectroscopy (ESI-MS/MS) detected addition of $\left[{ }^{13} \mathrm{C}_{U}\right]-\mathrm{Glc}$ units, then determined the molecular mass of the derivatized reducing end residue that was covalently labeled with the N-EDA tag. In these experiments, N-EDA mass label was attached uniquely to ${ }^{12} \mathrm{C}_{\mathrm{U}}$-glucose, meaning only pre-existing residues within maltohexaose, not newly added glucosyl units from the enzymatic reaction, were located at the reducing end (Larson et al., 2016). This provides direct evidence for non-reducing end addition by maize SSIIa. That conclusion was confirmed by ${ }^{13} \mathrm{C}$-NMR analyses that distinguished between free anomeric carbons and those bound in $\alpha-(1 \rightarrow 4)$ glycoside bonds. Anomeric carbons in newly incorporated residues were found to be glycoside-bound, not free, thus confirming non-reducing end addition (Larson et al., 2016).

The possibility remains that distinctions between SS classes could include different reaction mechanisms, and that SSs other than maize SSIIa could elongate glucan polymers at the reducing end. This is pertinent because the experiments supporting a reducing end mechanism used starch granules as a SS source to catalyze addition of new residues (Mukerjea et al., 2002; Mukerjea and Robyt, 2005a), and these contain multiple enzymes including SSI, SSII, and GBSS (Grimaud et al., 2008). To test whether nonreducing end addition is universal among SS classes, the current study characterized addition products formed by recombinant maize SSI. [ ${ }^{13} \mathrm{C}_{\mathrm{U}}$-Glc] labeling and product characterization by ESI-MS/MS revealed exclusive elongation at the non-reducing end. Thus, as expected from structural similarities among the enzymes, non-reducing end addition is highly likely to be universal among all SS classes. 


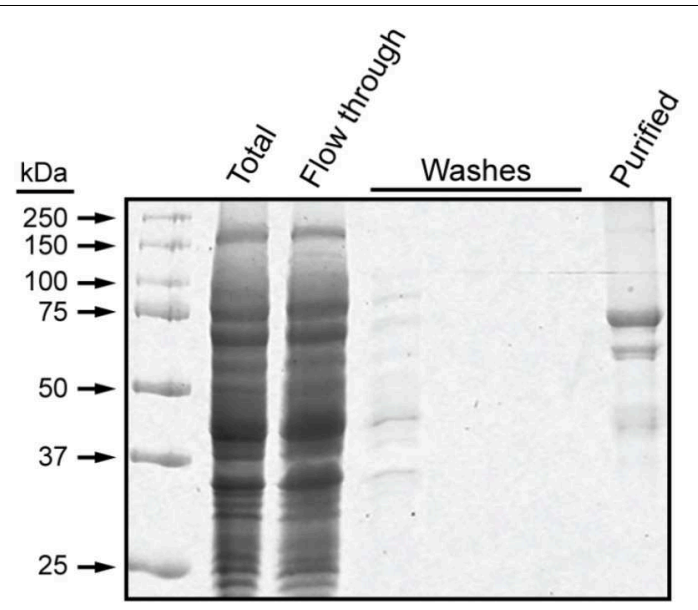

FIGURE 1 | NS-SSI purification. NS-SSI was purified from total soluble E. coli extract by binding to S-protein agarose. Proteins in each fraction were visualized by SDS-PAGE and Coomassie blue staining. The purified fraction was analyzed after boiling of the affinity matrix in SDS-PAGE loading buffer.

\section{MATERIALS AND METHODS}

\section{Recombinant SSI Expression}

Recombinant maize SSI was expressed from plasmid pET29NStagSSI (Huang et al., 2016). The pET-29 vector backbone provides the phage T7 promoter, and an E. coli ribosome binding site prior to the coding region. The expressed protein, designated NS-SSI, contains the 15 amino acid S-tag sequence at its $\mathrm{N}$ terminus followed by the mature maize SSI coding sequence. Expression of recombinant protein in E. coli Rosetta (DE3) pLys by induction of phage T7 polymerase with IPTG was as previously described. Briefly, cell pellets from $50 \mathrm{~mL}$ of induced culture were suspended in buffer A $(20 \mathrm{mM}$ Tris- $\mathrm{HCl}, \mathrm{pH} 7.5$, $150 \mathrm{mM} \mathrm{NaCl}$ ) supplemented with $0.1 \mathrm{mg} / \mathrm{mL}$ lysozyme, $5 \mathrm{mM}$ DTT, $1 \mathrm{mM}$ PMSF. Cells were lysed by sonication, then lysates were cleared by centrifugation and filtration through a $0.45 \mu \mathrm{m}$ syringe filter. NS-SSI was purified from cleared lysates by affinity to S-protein agarose equilibrated in buffer A. After binding, matrix with bound NS-SSI, referred to as "SSI beads," was washed extensively in buffer A and stored on ice. SSI beads were used directly as the catalyst in enzymatic reactions, within 2 days of their preparation.

Protein concentration on SSI beads was estimated after incubating $10 \mu \mathrm{L}$ samples of a 1:1 bead:buffer A slurry (v:v) with $20 \mu \mathrm{L}$ of $8 \mathrm{M}$ urea to release bound ligand. Beads were removed by centrifugation in a microfuge, then the $A_{280}$ of the supernatant was measured using a NanoDrop spectrophotomer. Protein concentration was calculated from the molar extinction coefficient of $112,760 \mathrm{~mol}^{-1} \mathrm{~cm}^{-1}$ predicted for the NS-SSI amino acid sequence. Typical purifications yielded approximately $15 \mu \mathrm{g}$ protein per $2 \mu \mathrm{L}$ of SSI bead slurry.

\section{Enzyme Assays}

Reactions were incubated at $30^{\circ} \mathrm{C}$ in $100 \mu \mathrm{L}$ final volume of $100 \mathrm{mM}$ bicine- $\mathrm{NaOH}, \mathrm{pH}$ 8.0, $5 \mathrm{mM}$ EDTA, $0.5 \mathrm{mg} / \mathrm{mL}$ BSA,
$5 \mathrm{mM}$ DTT. Rate measurements were from assays including $2 \mu \mathrm{L}$ of SSI bead slurry (approximately $15 \mu \mathrm{g}$ NS-SSI). Preliminary analyses with excess ADPGlc and glycogen determined that such reactions were rate-limited for enzyme. For kinetic analyses, glycogen concentration was constant at $10 \mathrm{mg} / \mathrm{mL}$ and ADPGlc concentration varied from 0.2 to $2.0 \mathrm{mM}$. Rate measurements using maltooligosaccharides were from reactions containing

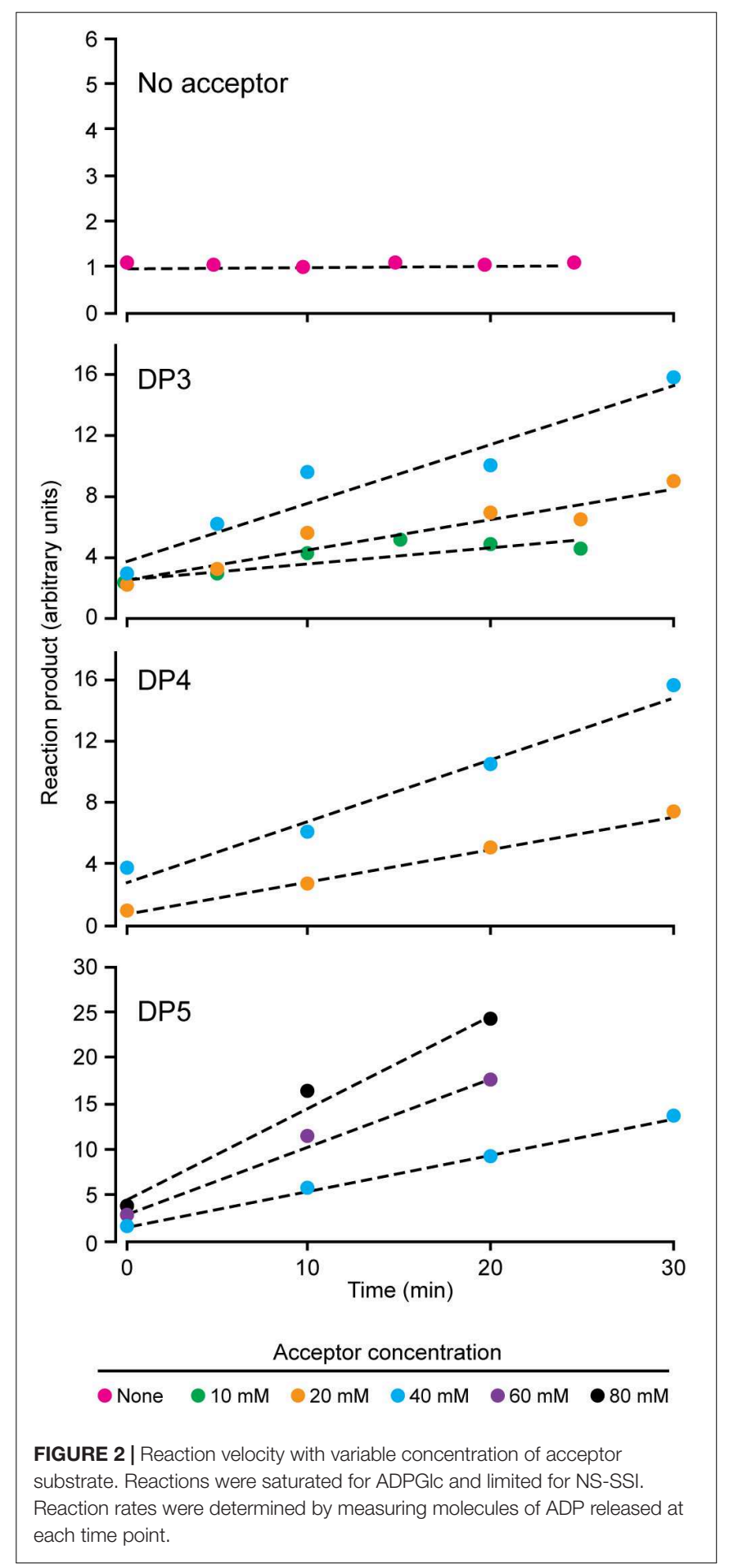




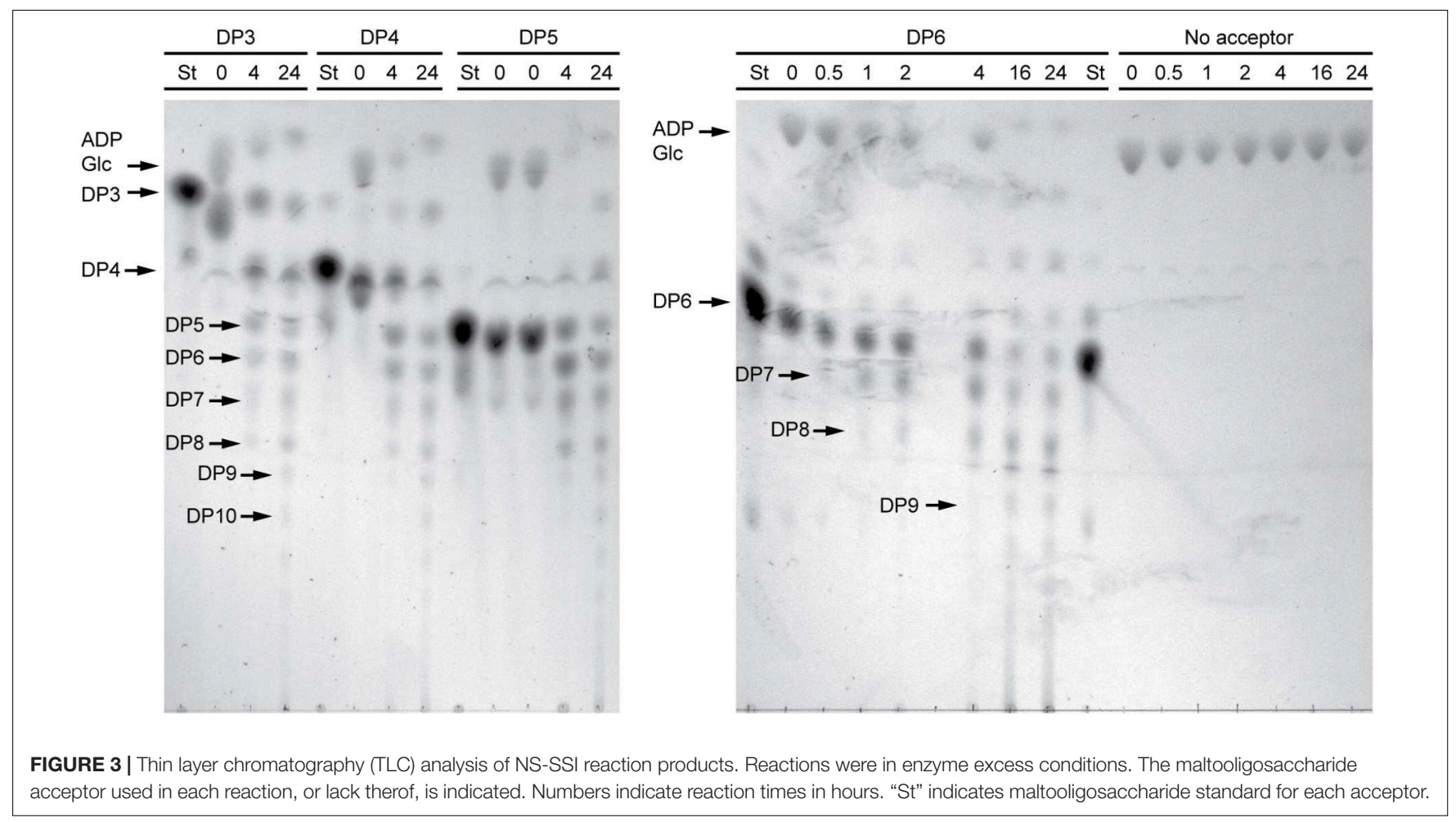

$2 \mathrm{mM}$ ADPGlc, with acceptor concentration varying from 10 to $80 \mathrm{mM}$. Reactions were initiated by addition of SSI beads and terminated at various times up to $30 \mathrm{~min}$ by boiling for $1 \mathrm{~min}$. Beads were removed by brief centrifugation and ADP product in $5 \mu \mathrm{L}$ of the supernatant was quantified using the ADP Glo Kinase Assay Kit (Promega No. V6930) as described previously (Huang et al., 2016; Larson et al., 2016).

Other reactions were carried out in conditions of enzyme excess, using $\left[{ }^{13} \mathrm{C}_{\mathrm{U}}\right]$-ADPGlc prepared as previously described (Larson et al., 2016). These "enzyme excess" reactions varied from the kinetic analyses in that they contained $50 \mu \mathrm{L}$ of SSI bead slurry, $4 \mathrm{mM}\left[{ }^{13} \mathrm{C}_{\mathrm{U}}\right]$-ADPGlc, and $2 \mathrm{mM}$ maltooligosaccharide, either maltotriose (DP3), maltotetraose (DP4), or maltopentaose (DP5). These reactions were incubated from 1 to $24 \mathrm{~h}$.

\section{Product Analysis}

Products of enzyme excess reactions were analyzed by TLC, high pressure anion exchange chromatography with pulsed amperometric detection (HPAEC-PAD), and ESI-MS/MS. The TLC method was described previously (Mukerjea et al., 2010; Larson et al., 2016). For HPAEC-PAD analysis, terminated reaction supernatant was diluted 1:20 into $\mathrm{H}_{2} \mathrm{O}$ and filtered through a $0.2 \mu \mathrm{m}$ syringe filter, then $25 \mu \mathrm{L}$ was injected into a Dionex IC-5000 chromatography system. Separation was on a Dionex CarboPac PA-100 $4 \mathrm{~mm} \times 250 \mathrm{~mm}$ column with a CarboPac PA-100 $4 \mathrm{~mm} \times 50 \mathrm{~mm}$ guard column at a flow rate of $1 \mathrm{~mL} / \mathrm{min}$. Elution was in $0.1 \mathrm{M} \mathrm{NaOH}$ for $4 \mathrm{~min}$ followed by a $40 \mathrm{~min}$ gradient of $0-0.4 \mathrm{M}$ sodium acetate in $0.1 \mathrm{M} \mathrm{NaOH}$. For ESI-MS/MS analysis, reaction products were first derivatized at the reducing end by reductive amination with N-EDA. Methods and instrumentation for derivatization and subsequent ESI-MS/MS analysis were described previously (Larson et al., 2016). MS1 spectra were averaged over fractions eluting from 9.2 to $9.4 \mathrm{~min}$. MS2 spectra were averaged over all fractions.

\section{RESULTS}

\section{Purification and Characterization of Recombinant SSI}

These experiments utilized a recombinant form of maize SSI that contains the 15 amino acid S-tag sequence at the $\mathrm{N}$ terminus, as previously described (Huang et al., 2016). Residues 39640 of full-length SSI were included, corresponding precisely to those present in mature protein following transit peptide removal during import into amyloplasts (Knight et al., 1998). The recombinant protein, termed NS-SSI, was expressed in E. coli and affinity purified from the soluble phase by binding to S-protein agarose. The purified fraction contained a major protein of approximately $70 \mathrm{kDa}$, matching the predicted molecular mass of NS-SSI (Figure 1). A minor contaminant of approximately $45 \mathrm{kDa}$ was shown to be a fragment of SSI, by immunoblot using SSI-specific antiserum (data not shown). An unidentified minor contaminant protein of approximately $60 \mathrm{kDa}$ was also present in the purified fraction.

NS-SSI bound to S-agarose was characterized to ensure it exhibited expected, reproducible enzymatic characteristics. Assays in the presence of ADPGlc donor and various maltooligosaccharide or polysaccharide acceptors were initiated 


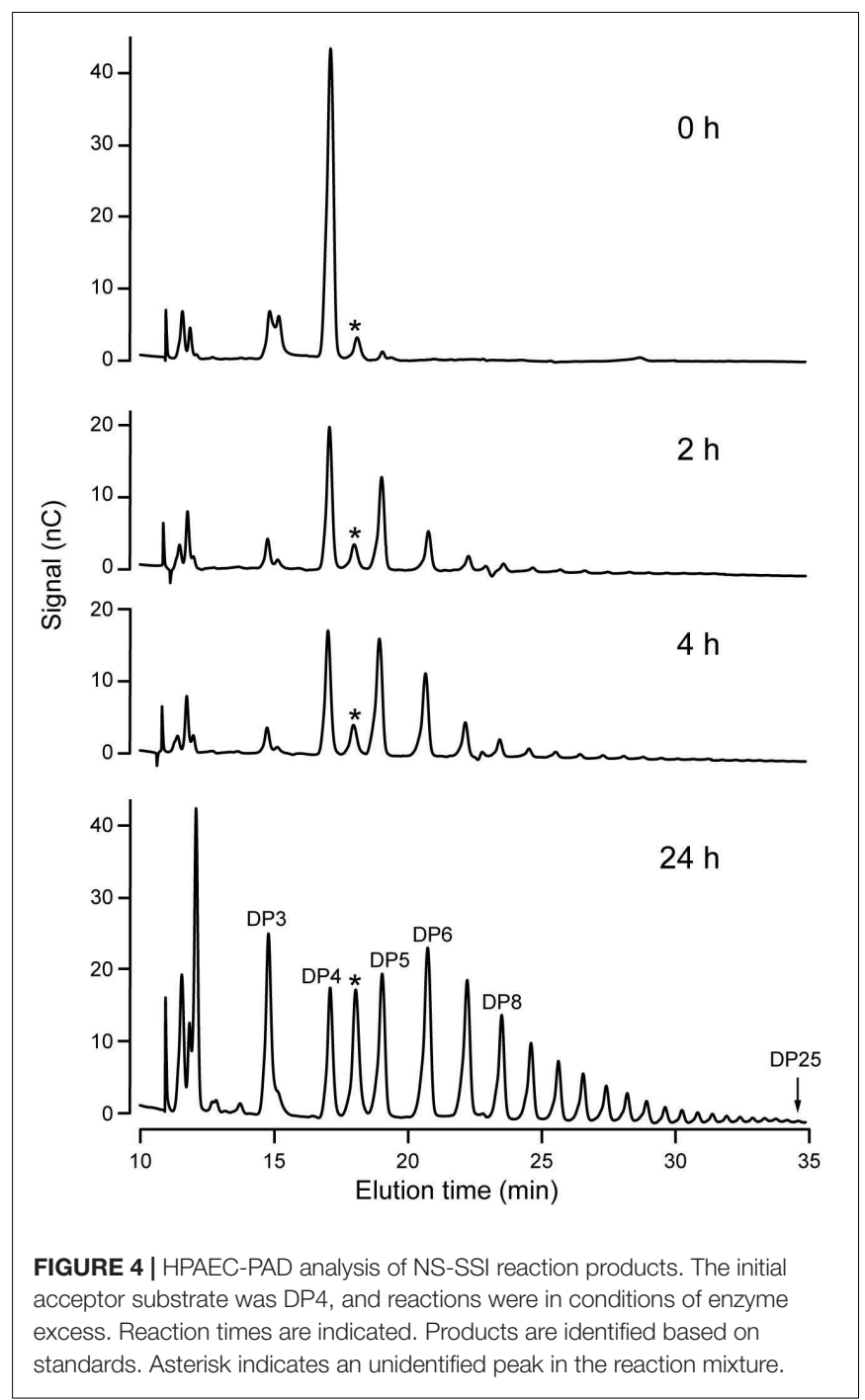

by addition of S-agarose-NS-SSI. Preliminary experiments ensured that reaction rates were linear with bead bed volume. Aliquots of the reaction mixture were removed at various times, catalysis was terminated by boiling, $\mathrm{S}$ agarose was removed by centrifugation, and ADP product was quantified by a commercial chemiluminescence assay. Kinetic analyses revealed the $\mathrm{Km}$ for ADPGlc, using either maltooligosaccharide or glycogen as the acceptor, to be approximately $0.5 \mathrm{mM}$. This value approximates the $\mathrm{Km}$ previously described for recombinant SSI from either maize or barley (Cuesta-Seijo et al., 2015; Huang et al., 2016). Holding ADPGlc concentration constant at $2 \mathrm{mM}$, approximately fourfold above the $\mathrm{Km}$, allowed testing of rates with variable concentrations of linear glucan acceptors, specifically, maltotriose (DP3), maltotetraose (DP4), and maltopentaose (DP5) (Figure 2). NS-SSI was active using any of these acceptors as the initial substrate. However, saturation of enzyme-substrate complex was never attained with any of the three glucans, even with up to $80 \mathrm{mM}$ acceptor in a reaction containing $<5 \mu \mathrm{M}$ enzyme. Such non-Michaelis-Menten behavior was observed previously for recombinant maize SSIIa (Larson et al., 2016), and recombinant SSI, SSII, and SSIII from barley (Cuesta-Seijo et al., 2015). In these in vitro conditions, NS-SSI required an acceptor glucan for activity (Figure 2).

\section{Enzyme Saturation Reactions}

In order to structurally analyze glucan products, reactions were run with excess enzyme to near complete depletion of ADPGlc, so that most of the acceptor substrate would be elongated. Product formation was observed by thin layer chromatography (TLC) (Figure 3). In these conditions NS-SSI was active on DP3, DP4, DP5, or DP6 to approximately the same extent. In each instance the majority of initial acceptor substrate molecules was converted to a mixture of longer polymers of increasing degrees of polymerization. Polymerization of glucosyl units was dependent on presence of an acceptor glucan, as shown by lack of higher molecular weight molecules when glucan was omitted from the reaction. Accordingly, ADPGlc was not consumed when incubated with NS-SSI in the absence of a glucan substrate.

Increased resolution of the products formed in the reaction was achieved using HPAEC-PAD. An example is shown using DP4 as the initial acceptor (Figure 4). Over a $24 \mathrm{~h}$ period, NSSSI catalyzed formation of a range of linear chains extending to at least DP25. At the last time point, glucans shorter than the initial substrate, specifically DP2 and DP3, were prevalent within the mixture of products. Removal of glucosyl residues is expected to result from the reverse reaction catalyzed by SS that occurs as ADPGlc is consumed and the concentration of ADP product increases. Reversible SS activity was demonstrated previously for recombinant forms of Arabidopsis and barley SSI (Brust et al., 2013; Cuesta-Seijo et al., 2015). Alternatively, the trivial explanation that a contaminant in the S-protein agarose fraction bound to NS-SSI possesses glucan hydrolase activity, has not been ruled out.

\section{Structural Characterization of Newly Polymerized Linear Glucans}

Elongation reactions catalyzed by NS-SSI in saturating conditions were performed using DP3, DP4, and DP5 with $\left[{ }^{13} \mathrm{C}_{\mathrm{U}}\right]$ ADPGlc as the donor substrate, in order to distinguish between newly added glucosyl units and pre-existing residues of the acceptor. After $4 \mathrm{~h}$ of reaction in excess enzyme conditions and limiting $\left[{ }^{13} \mathrm{C}_{\mathrm{U}}\right]-\mathrm{ADPGlc}$, reducing ends of the glucan products were labeled by reductive amination with $\mathrm{N}$-EDA. Molecules present in the labeling reactions were fractionated by reverse-phase chromatography, then subjected to electrospray ionization, and resulting ions were characterized by mass spectroscopy. Chromatographic fractions containing mass features matching the prediction for N-EDA-labeled initial substrate were identified. In reactions lacking enzyme these mass features were essentially pure (Figure 5A). Larger masses appeared in the spectra of reactions including NS-SSI that matched the prediction for N-EDA-labeled glucans elongated by one or two glucosyl units derived from $\left[{ }^{13} \mathrm{C}_{\mathrm{U}}\right]$-ADPGlc (Figure 5B). Reaction products containing fewer glucosyl units than the starting acceptor substrate were also detected in MS1 


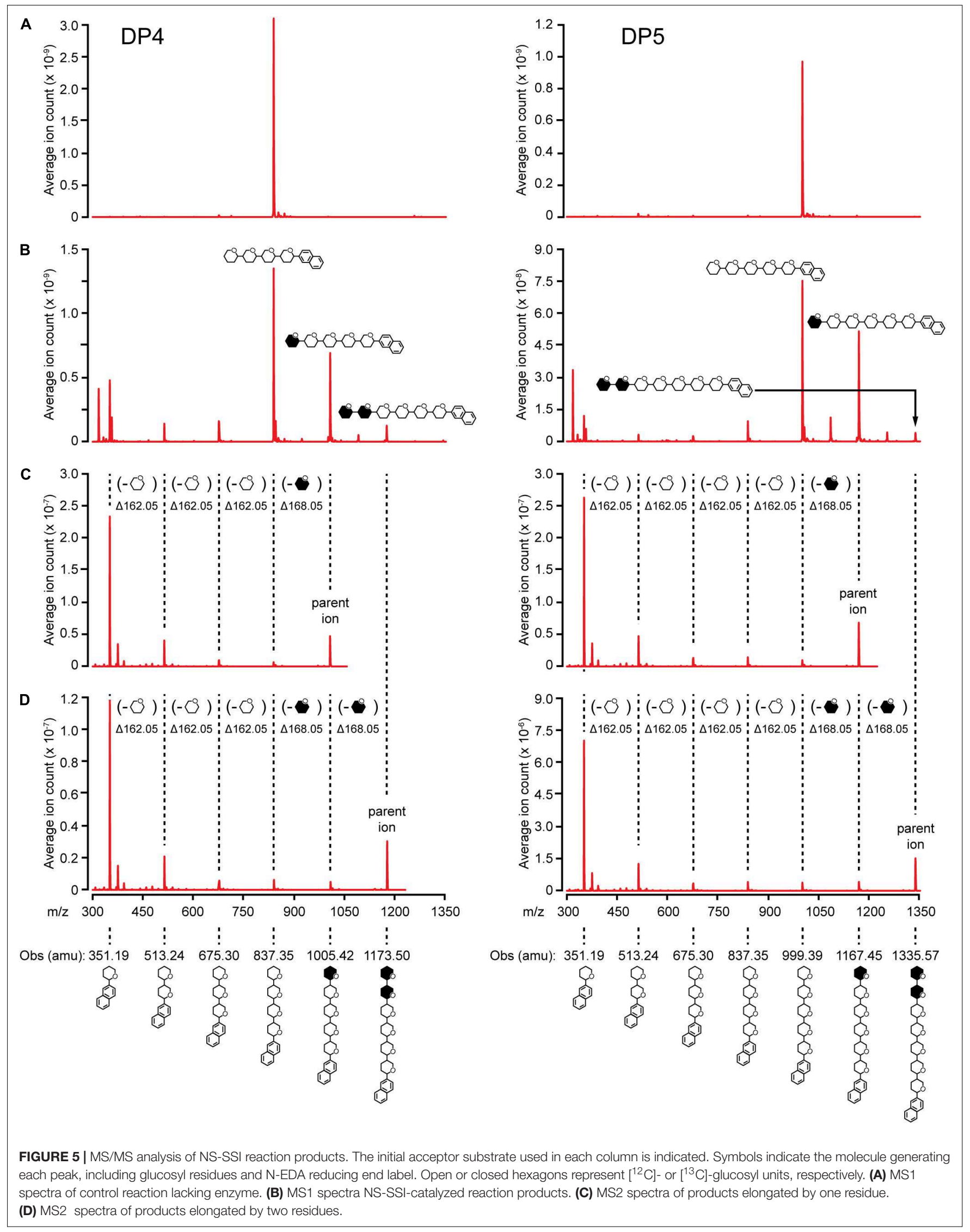


spectra. These presumably result from the SSI reverse reaction, as was also observed by HPAEC-PAD analysis (Figure 4).

${ }^{13} \mathrm{C}$-labeled glucosyl units potentially could have been added at either the non-reducing or reducing end. Analysis of mass fragmentation patterns collected by selecting then fragmenting the parent ions distinguished between these possibilities. The parent ion derived from extension of the substrate by one unit was subjected to further ionizing radiation and the masses of the resultant fragmentation ions were determined. If a newly incorporated $\left[{ }^{13} \mathrm{C}\right]$-labeled glucose residue were added at the reducing end, then derivatized with $\mathrm{N}$-EDA, the molecular mass of the fragment containing one glucose unit would be 357.19 Da. Alternatively, if new incorporations occur at the non-reducing end, then N-EDA would be linked to an $\left[{ }^{12} \mathrm{C}\right]$ glucosyl unit pre-existing in the acceptor substrate. In that instance the molecular mass of the fragment containing one $\left[{ }^{12} \mathrm{C}\right]$-glucosyl unit would be 351.19 Da. Fragments of 357.19 Da were never observed in the second dimension mass spectra, whereas fragments of 351.19 were prominent. These data indicate the acceptor substrate was elongated at the non-reducing end (Figures 5C,D).

Further, the difference in mass between the parent ion and the second dimension fragment that had lost one glucosyl unit could be determined. The unit that is removed must be at the non-reducing end, because all fragments in this analysis retain the N-EDA moiety located at the reducing end. This mass difference was $168.05 \mathrm{Da}$, indicative of a newly incorporated residue. Taken together the data provide conclusive evidence that glucosyl units derived from ADP-Glc, and added to glucan acceptors by NS-SSI action, are located at the non-reducing end.

\section{DISCUSSION}

The current study adds to previous characterization of the site of addition of new glucosyl units to growing $\alpha(1 \rightarrow 4)$-linked glucan polymers as catalyzed by SS. Together the data demonstrate definitively that recombinant SSI and SSIIa from maize both add glucose units at the non-reducing end. These results are fully consistent with the known structure of E. coli glycogen synthase bound to maltooligosaccharide acceptor. Prokaryotic glycogen synthases and the catalytic domain of plant SSs are conserved at the amino acid level, and both exhibit the GT-B structural fold (Sheng et al., 2009a; Cuesta-Seijo et al., 2013). The E. coli enzyme has been co-crystallized with maltohexaose, and that structure reveals three bound glucosyl units with the non-reducing residue opposed to the ADPGlc donor substrate (Sheng et al., 2009b). Taken together these data definitively eliminate the proposed mechanism in which glucosyl units are added at the reducing end of the acceptor. Further, the fact that both SSI and SSII have now

\section{REFERENCES}

Brust, H., Orzechowski, S., Fettke, J., and Steup, M. (2013). Starch synthesizing reactions and paths: in vitro and in vivo studies. J. Appl. Glycosci. 60, 3-20. doi: 10.5458/jag.jag.JAG-2012_018 been shown to utilize non-reducing end mechanisms indicates that all of the five conserved classes of this enzyme likely act in the same way.

The reasons that five classes of SS are conserved in the plant kingdom remain to be determined. However, alternative mechanisms of glucose unit addition in the various enzyme classes is highly unlikely to be part of the explanation. The current study further confirms the previous finding that evolutionary selection of SSI does not relate to limits on the degree of polymerization of the products, as had been proposed based on indirect measurement of affinity to branched glucans with varying chain length distributions (Commuri and Keeling, 2001). HPAEC-PAD characterization of NS-SSI products starting from maltotetraose showed the enzyme is capable of producing glucans of at least DP25. Previous data with recombinant barley SSI showed it capable of generating product of DP40 or more (Cuesta-Seijo et al., 2015). Thus, the SSI class in general is not inherently restricted to production of chains of DP12 or less, as could be predicted from genetic analyses showing accumulation of such chains when SSII is absent. The reasons for these observed alterations in amylopectin structure in mutant plant tissues, therefore, likely are related to in vivo factors that are not reproduced in recombinant systems.

\section{AUTHOR CONTRIBUTIONS}

YX, TH-B, and AM performed the experiments and analyzed the data. TH-B, AB, and AM conceived the experiments and analyzed the data. All authors contributed to drafting the manuscript and approved the final version.

\section{FUNDING}

This study was funded by the National Program on Key Basic Research Projects of China (973 program, grant no. 2014CB138202), award 1517256 from the National Science Foundation to AM, award 2017-67013-26189 from the NIFA, United States Department of Agriculture to AM and TH-B, and award R01-GM115489 from the NIGMS, National Institutes of Health to AB.

\section{ACKNOWLEDGMENTS}

The authors acknowledge Mr. Joel Nott and the Iowa State University Protein Facility for assistance with ESI-MS/MS analysis and providing mass spectrometry instrumentation and Dr. Daniel Falconer for providing expertise with TLC and ESIMS/MS procedures.

Commuri, P. D., and Keeling, P. L. (2001). Chain-length specificities of maize starch synthase I enzyme: studies of glucan affinity and catalytic properties. Plant J. 25, 475-486. doi: 10.1046/j.1365-313x.2001.00955.x

Crofts, N., Sugimoto, K., Oitome, N. F., Nakamura, Y., and Fujita, N. (2017). Differences in specificity and compensatory functions among three major starch 
synthases determine the structure of amylopectin in rice endosperm. Plant Mol. Biol. 94, 399-417. doi: 10.1007/s11103-017-0614-8

Cuesta-Seijo, J. A., Nielsen, M. M., Marri, L., Tanaka, H., Beeren, S. R., and Palcic, M. M. (2013). Structure of starch synthase I from barley: insight into regulatory mechanisms of starch synthase activity. Acta Crystallogr. D Biol. Crystallogr. 69, 1013-1025. doi: 10.1107/S090744491300440X

Cuesta-Seijo, J. A., Nielsen, M. M., Ruzanski, C., Krucewicz, K., Beeren, S. R., Rydhal, M. G., et al. (2015). In vitro biochemical characterization of all barley endosperm starch synthases. Front. Plant Sci. 6:1265. doi: 10.3389/fpls.2015. 01265

De Fekete, M. A., Leloir, L. F., and Cardini, C. E. (1960). Mechanism of starch biosynthesis. Nature 187, 918-919. doi: 10.1038/187918a0

Delvallé, D., Dumez, S., Wattebled, F., Roldán, I., Planchot, V., Berbezy, P., et al. (2005). Soluble starch synthase I: a major determinant for the synthesis of amylopectin in Arabidopsis thaliana leaves. Plant J. 43, 398-412. doi: 10.1111/j. 1365-313X.2005.02462.x

Deschamps, P., Moreau, H., Worden, A. Z., Dauvillee, D., and Ball, S. G. (2008). Early gene duplication within chloroplastida and its correspondence with relocation of starch metabolism to chloroplasts. Genetics 178, 2373-2387. doi: 10.1534/genetics.108.087205

Fujita, N., Yoshida, M., Asakura, N., Ohdan, T., Miyao, A., Hirochika, H., et al. (2006). Function and characterization of starch synthase I using mutants in rice. Plant Physiol. 140, 1070-1084. doi: 10.1104/pp.105.071845

Fujita, N., Yoshida, M., Kondo, T., Saito, K., Utsumi, Y., Tokunaga, T., et al. (2007). Characterization of SSIIIa-deficient mutants of rice: the function of SSIIIa and pleiotropic effects by SSIIIa deficiency in the rice endosperm. Plant Physiol. 144, 2009-2023. doi: 10.1104/pp.107.102533

Grimaud, F., Rogniaux, H., James, M. G., Myers, A. M., and Planchot, V. (2008). Proteome and phosphoproteome analysis of starch granule-associated proteins from normal maize and mutants affected in starch biosynthesis. J. Exp. Bot. 59, 3395-3406. doi: 10.1093/jxb/ern198

Hennen-Bierwagen, T. A., James, M. G., and Myers, A. M. (2012). "Involvement of debranching enzymes in starch biosynthesis," in Essential Reviews In Experimental Biology, The Synthesis and Breakdown of Starch, Vol. 5, ed. I. J. Tetlow (London: Society For Experimental Biology), 179-215.

Hennen-Bierwagen, T. A., and Myers, A. M. (2013). "Genomic specification of starch biosynthesis in maize endosperm," in Seed Genomics, ed. P. Becraft (Ames, IA: Wiley-Blackwell), 123-137. doi: 10.1002/9781118525524.ch7

Huang, B., Keeling, P. L., Hennen-Bierwagen, T. A., and Myers, A. M. (2016). Comparative in vitro analyses of recombinant maize starch synthases SSI, SSIIa, and SSIII reveal direct regulatory interactions and thermosensitivity. Arch. Biochem. Biophys. 596, 63-72. doi: 10.1016/j.abb.2016.02.032

Jeon, J.-S., Ryoo, N., Hahn, T.-R., Walia, H., and Nakamura, Y. (2010). Starch biosynthesis in cereal endosperm. Plant Physiol. Biochem. 48, 383-392. doi: 10.1016/j.plaphy.2010.03.006

Keeling, P. L., and Myers, A. M. (2010). Biochemistry and genetics of starch synthesis. Annu. Rev. Food Sci. Technol. 1, 271-303. doi: 10.1146/annurev.food. 102308.124214

Knight, M. E., Harn, C., Lilley, C. E., Guan, H., Singletary, G. W., Muforster, C., et al. (1998). Molecular cloning of starch synthase I from maize (W64) endosperm and expression in Escherichia coli. Plant J. 14, 613-622. doi: 10.1046/ j.1365-313X.1998.00150.x

Larson, M. E., Falconer, D. J., Myers, A. M., and Barb, A. W. (2016). Direct characterization of the maize starch synthase IIa product shows maltodextrin elongation occurs at the non-reducing end. J. Biol. Chem. 291, 24951-24960. doi: 10.1074/jbc.M116.754705

Leloir, L. F., De Fekete, M. A., and Cardini, C. E. (1961). Starch and oligosaccharide synthesis from uridine diphosphate glucose. J. Biol. Chem. 236, 636-641.
Mukerjea, R., Falconer, D. J., Yoon, S. H., and Robyt, J. F. (2010). Large-scale isolation, fractionation, and purification of soluble starchsynthesizing enzymes: starch synthase and branching enzyme from potato tubers. Carbohydr. Res. 345, 1555-1563. doi: 10.1016/j.carres.2010. 04.022

Mukerjea, R., and Robyt, J. F. (2005a). Starch biosynthesis: further evidence against the primer nonreducing-end mechanism and evidence for the reducing-end two-site insertion mechanism. Carbohydr. Res. 340, 2206-2211.

Mukerjea, R., and Robyt, J. F. (2005b). Starch biosynthesis: the primer nonreducing-end mechanism versus the nonprimer reducing-end two-site insertion mechanism. Carbohydr. Res. 340, 245-255.

Mukerjea, R., and Robyt, J. F. (2012). De novo biosynthesis of starch chains without a primer and the mechanism for its biosynthesis by potato starch-synthase. Carbohydr. Res. 352, 137-142. doi: 10.1016/j.carres.2011.11.024

Mukerjea, R., Yu, L., and Robyt, J. F. (2002). Starch biosynthesis: mechanism for the elongation of starch chains. Carbohydr. Res. 337, 1015-1022. doi: 10.1016/ S0008-6215(02)00067-8

Nakamura, Y. (2015). “Biosynthesis of reserve starch," in Starch, ed. Y. Nakmura (Berlin: Springer), 161-209.

Recondo, E., and Leloir, L. F. (1961). Adenosine diphosphate glucose and starch synthesis. Biochem. Biophys. Res. Commun. 6, 85-88. doi: 10.1016/0006291X(61)90389-8

Sheng, F., Jia, X., Yep, A., Preiss, J., and Geiger, J. H. (2009a). The crystal structures of the open and catalytically competent closed conformation of Escherichia coli glycogen synthase. J. Biol. Chem. 284, 17796-17807. doi: 10.1074/jbc. M809804200

Sheng, F., Yep, A., Feng, L., Preiss, J., and Geiger, J. H. (2009b). Oligosaccharide binding in Escherichia coli glycogen synthase. Biochemistry 48, 10089-10097. doi: 10.1021/bi900916t

Szydlowski, N., Ragel, P., Raynaud, S., Lucas, M. M., Roldan, I., Montero, M., et al. (2009). Starch granule initiation in Arabidopsis requires the presence of either class IV or class III starch synthases. Plant Cell 21, 2443-2457. doi: 10.1105/tpc.109.066522

Zeeman, S. C., Kossmann, J., and Smith, A. M. (2010). Starch: its metabolism, evolution, and biotechnological modification in plants. Annu. Rev. Plant Biol. 61, 209-234. doi: 10.1146/annurev-arplant-042809-112301

Zhang, X., Colleoni, C., Ratushna, V., Sirghie-Colleoni, M., James, M. G., and Myers, A. M. (2004). Molecular characterization demonstrates that the Zea mays gene sugary 2 codes for the starch synthase isoform SSIIa. Plant Mol. Biol. 54, 865-879. doi: 10.1007/s11103-004-0312-1

Zhu, F., Bertoft, E., Myers, A. M., and Seetharaman, K. (2013). Molecular structure of starches from maize mutants deficient in starch synthase III. J. Agric. Food Chem. 61, 9899-9907. doi: 10.1021/jf402090f

Conflict of Interest Statement: The authors declare that the research was conducted in the absence of any commercial or financial relationships that could be construed as a potential conflict of interest.

The reviewer IT and handling Editor declared their shared affiliation.

Copyright (c) 2018 Xie, Barb, Hennen-Bierwagen and Myers. This is an open-access article distributed under the terms of the Creative Commons Attribution License (CC BY). The use, distribution or reproduction in other forums is permitted, provided the original author(s) and the copyright owner(s) are credited and that the original publication in this journal is cited, in accordance with accepted academic practice. No use, distribution or reproduction is permitted which does not comply with these terms. 